\title{
NOMINATA DE PARECERISTAS
}

Dossiê Educação e linguagem na sociedade digital: crítica e construção de sentidos

Número 15 (Maio-Ago. 2019)

Adriana Lúcia Escobar Chaves de Barros

Universidade Estadual de Mato Grosso do Sul

Inés Kayon de Miller

Pontifícia Universidade Católica do Rio de Janeiro

Katia Nazareth Moura de Abreu

Universidade do Estado do Rio de Janeiro

Marcia Lisbôa Costa de Oliveira

Universidade do Estado do Rio de Janeiro

Nara Hiroko Takaki

Universidade Federal do Mato Grosso do Sul

Ricardo Toshihito Saito

Universidade Federal da Bahia

Ruberval Franco Maciel

Universidade Estadual de Mato Grosso do Sul

Sandra Regina Buttros Gattolin de Paula

Universidade Federal de São Carlos

Simone Batista da Silva

Universidade Federal Rural do Rio de Janeiro

Souzana Mizan

Universidade Federal de São Paulo

Valéria Campos Muniz

Instituto Nacional de Educação de Surdos

Vera Teixeira da Silva

Universidade do Estado do Rio de Janeiro 